\title{
No sample left behind
}

\section{Maximizing the clinical impact of immune profiling will require dedicated immune monitoring facilities and community standards for handling patient samples.}

ast month's Nature conference, "Immune Profiling in Health and LDisease," organized in Seattle with Adaptive Biotechnologies, showcased high-throughput technologies, such as next-generation sequencing and mass cytometry, that are transforming our understanding of the human immune system. These approaches are providing insights into the transcriptomes, methylomes, and T cell receptor (TCR) and B cell receptor (BCR) repertoires of patients in trials of a raft of new immune therapies. They are helping to unravel how and why these patients respond differently to cancer immunotherapy-and increasingly to other interventions, such as vaccination, autoimmune treatments and transplantation. And yet, despite this rapid progress, the reality is that most immune profiling efforts remain at a pilot scale. To truly galvanize clinically actionable insights, researchers will need to integrate datasets of sufficient diversity and statistical power. This will require greater attention to how samples are acquired and analyzed and community agreement on how store, share and interpret data.

Cancer immunotherapy has been all the rage of late; indeed, just a few weeks ago, Jim Allison received the Lasker prize for "the discovery and development of a monoclonal antibody therapy that unleashes the immune system to combat cancer." Although clinical data from checkpoint inhibitors and adoptive $\mathrm{T}$ cell therapy are promising, our understanding of the underlying human immune biology remains rudimentary. Questions, such as which therapeutic combinations make the most sense for a specific tumor type, which therapies generate desirable signatures of $\mathrm{T}$ cell memory and which generate broadly neutralizing antibodies, remain active areas of investigation. Only about one in five melanoma patients respond to cytotoxic T lymphocyte antigen (CTLA)-4 blockade. In this context, it is important to understand how we can determine a priori who will benefit from treatment and who will not.

As the science on show at the Seattle event demonstrated, we now have a range of technologies to answer these questions. T and B cell receptor repertoires can be mapped, receptors cloned, neoepitopes identified, immune cells characterized at the level of the genome, transcriptome, methylome, proteome and metabolome, and dozens of cell surface markers and cytokines monitored.

The problem is that most immune-profiling analyses remain rather limited in scope.

This is understandable because in many cases, samples are acquired for specific purposes, such as tumor biopsies for diagnosis or blood draws for determining tumor burden. Once a sample has been used to answer a research question, often the remaining tissue or cell sample is lost. Samples often have a single and final owner-an individual who writes a protocol with a specific purpose in mind. To make matter worse, in industrysponsored studies, samples often remain sequestered in company freezers.

Funding mechanisms don't always help. Sample acquisition, storage and analysis are often covered in grants only as an afterthought. Insurance companies pay only for a few US Food and Drug Administration-approved assays. Drug companies have little incentive to fund unsupervised analyses of their patient cohorts. Grants focus on an investigator's one-dimensional analysis of samples and fail to provide funding for sample studies beyond that analysis. Identifying sources that will pay for assays and banking costs that may not yield immediate results is often nontrivial. In rare cases, an academic center may come to the rescue, but institutional support is often a hard-fought gain.

Setting up a comprehensive immune profiling facility at an academic center is no mean feat. Such efforts must decide which assays should be run on solid tumor biopsies or on liquid tumors; which antibodies should be used against which markers; which types of analyte (for example, DNA, RNA, protein) should be isolated; which technology should be used to interrogate the sample; and how a sample should be dissociated, cryopreserved or fixed with formalin.

Some institutions, such as Stanford University, the Icahn School of Medicine at Mount Sinai and the MD Anderson Cancer Center, are showing the way. At Stanford, patient samples from diverse trials and protocols have been collected systematically for almost a decade. A set of standard assays is run on fresh samples as they come in, and these data provide information that is used to annotate each sample. The annotation allows the bank to be easily searchable and immediately available-when a researcher needs samples with particular characteristics to test a new hypothesis, they can be identified without delay. The rest of the material is processed for storage using consistent protocols.

Institutions that set up such centers provide a boon for internal faculty. But what if such facilities could collaborate and facilitate the pooling of data between institutions?

Certainly, it is unlikely that institutions will share samples. But if appropriate informed consent is obtained at the outset, there is no reason why anonymized annotated data on these samples could not be shared.

To achieve this, there needs to be agreement between institutions on best practices for running assays on different sample types and on how to share data. Stand Up To Cancer's inter-institutional effort involving Massachusetts General Hospital Cancer Center, Memorial Sloan Kettering and MD Anderson, among others, is an example of just such a collaboration.

But for different datasets to be comparable, there also needs to broad community agreement not only on the minimum set of information required to describe sample acquisition, the methodology employed and data analysis carried out, but also on the standardized formats for depositing and exchanging data. In this respect, a meeting late last May suggests that the community is beginning to tackle these issues (http:// bit.ly/1gyKXt3).

For the moment, however, cross-insitutional efforts remain the exception rather than the rule. For immune profiling research to truly fulfill its potential, now is the time for the community to come together and agree on standardized sample collection and storage and the benefits of pooling data. We must open up the freezers and unlock the data. No sample should be left behind. 